POPULATION STUDY ARTICLE

\title{
Outcomes of singleton small for gestational age preterm infants exposed to maternal hypertension: a retrospective cohort study
}

\author{
Elhaytham ElSayed ${ }^{1}$, Sibasis Daspal ${ }^{2}$, Wendy Yee ${ }^{1}$, Ermelinda Pelausa ${ }^{3}$, Rody Canning ${ }^{4}$, Prakesh S. Shah ${ }^{5}$ and \\ Kamran Yusuf (iD) on behalf of the Canadian Neonatal Network Investigators
}

BACKGROUND: Hypertensive disorders of pregnancy (HDP) are a major cause of small for gestational age (SGA). Preterm SGA infants have higher rates of adverse outcomes than appropriate for gestational age infants. However, the outcomes are not well established in the setting of HDP.

METHODS: Retrospective population-based study using the Canadian Neonatal Network database from January 1, 2010 to December 31, 2016 of SGA infants $<33$ weeks gestation. Using multivariable models, we determined the adjusted odds ratios (AORs) with $95 \%$ confidence intervals (CI) for mortality, bronchopulmonary dysplasia (BPD), severe intraventricular hemorrhage (IVH), severe retinopathy of prematurity, necrotizing enterocolitis, late-onset sepsis, and patent ductus arteriosus (PDA) in infants of HDP mothers and compared them to infants of non-HDP mothers.

RESULTS: Of the 2081 eligible SGA infants, 1317 (63\%) were born to HDP mothers and had lower odds of mortality (AOR 0.57, 95\% $\mathrm{Cl} 0.39-0.83$ ) and BPD (AOR 0.69, 95\% Cl 0.53-0.90). Sub-group analysis demonstrated decreased mortality in 26-28 and 29-32 weeks gestation groups, decreased BPD in 29-32 weeks gestation group, and decreased PDA in <26 weeks gestation group. CONCLUSION: Preterm SGA infants of HDP mothers have lower odds of mortality and BPD compared to infants of non-HDP mothers.

Pediatric Research (2019) 86:269-275; https://doi.org/10.1038/s41390-019-0416-6

\section{INTRODUCTION}

Preterm infants with intrauterine growth restriction are at higher risk of neonatal mortality and morbdity than appropriate for gestational age (AGA) infants., ${ }^{1,2}$ Although different measures of intrauterine growth are used in studies of growth-restricted infants, most investigations have used small for gestational age (SGA), defined as birthweight $<10$ th percentile for gestational age and sex, as a marker for fetal growth restriction. In the 1970s and 1980s, SGA infants were considered to be at lower risk of adverse neonatal outcomes, especially respiratory outcomes. Accelerated pulmonary maturation in response to intrauterine stress was believed to be responsible for the lowered risk of adverse outcomes. ${ }^{2}$ More recent data, however, suggests that SGA is a major risk factor for adverse neonatal outcomes. Increased mortality and bronchopulmonary dysplasia (BPD) in SGA infants is seen consistently across studies on SGA infants. ${ }^{3}$ However, reports of other outcomes are variable. Some studies have reported higher rates of necrotizing enterocolitis (NEC), retinopathy of prematurity (ROP), sepsis, and intraventriculat hemorrhage (IVH) in SGA infants compared to AGA infants and some have not. ${ }^{1-6}$ Poor neurodevelopmental outcome is also associated with SGA infants. ${ }^{7}$ These inconsistencies may be due to different definitions of SGA, study methodologies, sample sizes, and gestational age and birthweight ranges. However, a major reason for these discrepant results may be because most studies on outcomes of preterm SGA infants have compared them to AGA infants, treating SGA infants as a homogeneous group despite
SGA having several different causes. ${ }^{8}$ Although gestational age and birthweight are the most important risk factors for poor outcomes in preterm infants, pregnancy complications that affect fetal growth also play an important role. ${ }^{4}$ Research on outcomes of SGA infants has paid little attention to the role of pregnancy complications that affect fetal growth. One common pregnancy complication associated with SGA is the hypertensive disorders of pregnancy (HDP).

HDP, comprising preeclampsia, gestational hypertension, chronic hypertension, and preeclampsia superimposed on chronic hypertension, are a significant global health problem, affecting $5-8 \%$ of all pregnancies. ${ }^{9}$ In industrialized countries, HDP are a major cause of maternal mortality and increased rates of stillbirth and neonatal death at all gestational ages. ${ }^{9}$ In addition, preterm infants born to mothers with HDP have an increased risk of several morbidities, including respiratory distress syndrome (RDS), BPD, and ROP. ${ }^{10,11}$ Some studies have demonstrated a lower rate of IVH and higher rates of sepsis and NEC in infants of mothers with HDP, while others have demonstrated no difference. ${ }^{11}$ Furthermore, with the rising prevalence of predisposing factors such as increasing maternal age, obesity, assisted reproductive technologies, and diabetes, the worldwide incidence of HDP is increasing. ${ }^{9}$ Given that HDP affects fetal growth and is associated with neonatal morbidities, the aim of our study was to compare the outcomes of preterm SGA infants $<33$ weeks gestation born to hypertensive mothers with infants born to non-HDP mothers using the Canadian Neonatal Network (CNN) database.

\footnotetext{
${ }^{1}$ Department of Pediatrics, Cumming School of Medicine, University of Calgary, Calgary, AB, Canada; ${ }^{2}$ Department of Pediatrics, University of Saskatchewan, Saskatoon, SK, Canada; ${ }^{3}$ Department of Pediatrics, McGill University, Montreal, QC, Canada; ${ }^{4}$ Department of Pediatrics, University of New Brunswick, Saint John, NB, Canada and ${ }^{5}$ Department of Pediatrics, Mount Sinai Hospital and University of Toronto, Toronto, ON, Canada

Correspondence: Kamran Yusuf (kyusuf@ucalgary.ca)

Members of the Canadian Neonatal Network can be found in the Supplemental Authors List (online).
}

Received: 6 November 2018 Revised: 11 April 2019 Accepted: 18 April 2019

Published online: 13 May 2019 


\section{METHODS}

Study population

The CNN comprises all level III neonatal intensive care units (NICUs) in Canada and maintains an electronic national database of admissions. Maternal and neonatal anonymized data with standardized definitions are entered electronically at each site by trained abstractors into a customized data entry program with inherent error checks. Details of data collection and management have been previously published. ${ }^{12}$ The database has been validated with a high degree of precision, internal consistency, and reproducibility. ${ }^{13}$ The study population comprised singleton SGA infants $<33$ weeks gestation admitted to participating CNN NICUs between January 1, 2010 and December 31, 2016. Infants with missing data on maternal hypertension, moribund infants, and infants with congenital anomalies were excluded. Eligible infants were divided into two groups: infants with a maternal history of HDP and infants with non-HDP mothers. The Research Ethics Board of the University of Calgary (REB 17-1943) and the Steering Committee of the CNN approved the study.

\section{Definitions}

Variables were defined as per the CNN Abstractor's Manual. ${ }^{14}$ SGA was defined as birthweight below the 10th percentile for gestational age and sex according to Canadian standards. ${ }^{15}$ HDP were defined as hospital or in-office systolic blood pressure $\geq 140$ $\mathrm{mmHg}$ and/or diastolic blood pressure $\geq 90 \mathrm{mmHg}$, based on the average of at least two measurements from the same arm at least 15 min apart. ${ }^{10}$ HDP included pre-pregnancy hypertension, pregnancy-induced hypertension (PIH) (hypertension without proteinuria), preeclampsia, eclampsia, and preeclampsia superimposed on chronic hypertension. ${ }^{10}$ Data on maternal smoking were based on maternal self-report, abstracted from the maternal prenatal records, and were defined as any cigarette smoking during pregnancy. Duration of smoking and number of cigarettes smoked are not collected as part of the CNN database. Substance abuse, also based on maternal self-reporting, included use of all recreational drugs such as marijuana, heroin, codeine, and methadone. Antenatal steroid use was classified as any steroid use prior to birth and complete course of steroids. Diabetes included both gestational and pre-gestational forms of the disease. Gestational age was assessed in the following order of preference: date of in vitro fertilization, first trimester ultrasonography, last menstrual period, obstetric estimate, and pediatric estimate. The Score for Neonatal Acute Physiology (SNAP)Version II was calculated to assess illness severity on admission. Preterm premature rupture of membranes was defined as rupture of membranes more than $24 \mathrm{~h}$ prior to birth.

BPD was defined as supplemental oxygen use at 36 weeks corrected gestation or at discharge to a level I or II center. ${ }^{16}$ Severe IVH was defined as grade 3 or 4 according to the criteria of Papile. $^{17}$ Periventricular leukomalacia was diagnosed on head ultrasound findings after 21 days of life. NEC was defined according to the criteria of Bell and included infants with $\geq$ stage $2{ }^{18}$ ROP was defined according to the International Classification of ROP and included infants with $\geq$ stage 3 in either eye. ${ }^{19}$ Lateonset sepsis (LOS) was defined as the presence of a pathogenic organism in either blood or cerebrospinal fluid culture after $48 \mathrm{~h}$ of age. Patent ductus arteriosus (PDA) was identified based on clinical features and/or echocardiography.

\section{Outcomes}

In addition to mortality, outcomes included the following major morbidities in extremely preterm infants: BPD, grade 3 and $4 \mathrm{IVH}$, ROP $\geq$ stage 3, NEC, LOS, and clinically significant PDA. We also analyzed length of hospital stay, duration of supplemental oxygen use, duration of mechanical ventilation, and continuous positive airway pressure (CPAP) as markers of NICU resource use, and compared them between the two groups.

\section{Statistics}

Infant and maternal characteristics were analyzed using the $x^{2}$ test for categorical variables and the $t$ test or the Mann-Whitney $U$ test for continuous variables. For neonatal outcomes, odds ratios were calculated using logistic regression models adjusting for possible confounders, including maternal age, any antenatal steroid use, maternal smoking, gestational age, gender, cesarean section, out born status, and SNAP II scores $>20$. The outcomes studied were also analyzed in three sub-groups $(<26,26-28$, and $29-32$ weeks gestation). The interaction between gestational age groups and HDP was tested first and then sub-group analysis was performed for those outcomes with significant interaction terms. All duration variables (hospital stay, supplemental oxygen use, mechanical ventilation, CPAP use) were non-normally distributed. They were log transformed to fit linear regression models in order to adjust for confounders as above. The difference between the two groups was expressed as the ratio of two geometric means and was transformed back to the original scale for reporting. Non-HDP group was used as the reference for the percentage change. A two-sided $p$ value $<0.05$ was used to determine statistical significance. Data analysis was conducted using SAS v 9.3 (SAS Institute Inc., Cary, NC, USA)

\section{RESULTS}

Of the 30,223 infants, $<33$ weeks gestation admitted to participating NICUs during the study period, 1317 (4.3\%) SGA infants were born to HDP mothers and 764 (2.5\%) SGA infants were born to non-HDP mothers (Fig. 1). The rates of SGA infants of HDP and non-HDP mothers remained stable during the study period without any significant change (Fig. 2).

Table 1 shows the maternal and infant characteristics of the two study groups. In the HDP group, maternal age was higher with more primigravida and significantly higher antenatal steroid use and diabetes. In the non-HDP group, rates of smoking, substance abuse, and chorioamnionitis were higher with more males and out born infants. The gestational age and birthweight were similar between the two groups, but there were more infants in the 26-28 weeks gestation group than in the HDP group. One hundred and eighty-six (9\%) of infants were $<26$ weeks gestation. Although the proportion of infants with Apgar score $<7$ was similar between the two groups, the proportion of infants with SNAP II score $>20$ was higher in the non-HDP group. More infants in the HDP group required surfactant.

Table 2 shows the adjusted odds ratio (AOR) with 95\% confidence intervals $(\mathrm{Cl})$ of the outcomes in the whole cohort. Compared to the non-HDP group, the odds of mortality and BPD were significantly lower in the HDP group. There was no difference in the odds of severe ROP, NEC, LOS, or PDA between the two groups. Except for a longer duration of CPAP use in the HDP group, there was no difference in other markers of NICU resource use between the two groups (Table 3 ).

Significant interactions were observed between HDP exposure and gestational age groups for the various outcomes (Table 4). Although mortality was lower in all three gestational age groups for the HDP cohort, it was significant in the 26-28 and 29-32 weeks gestation groups only, with significant interaction between HDP and the gestational age sub-groups. The lower odds of BPD in the HDP group were significant only in the 29-32 weeks gestation group. Severe IVH was lower in all three gestational age groups for the HDP group, but was not statistically significant in any of the gestational age groups with non-significant interaction between HDP and the gestational age sub-groups. For all three gestational age groups, there was no difference between the two groups in the odds for severe ROP, NEC, or LOS. HDP infants in the $<26$ weeks gestational age group had lower odds of PDA, but not in the other two gestational age groups with significant interaction between HDP and the gestational age sub-groups. 


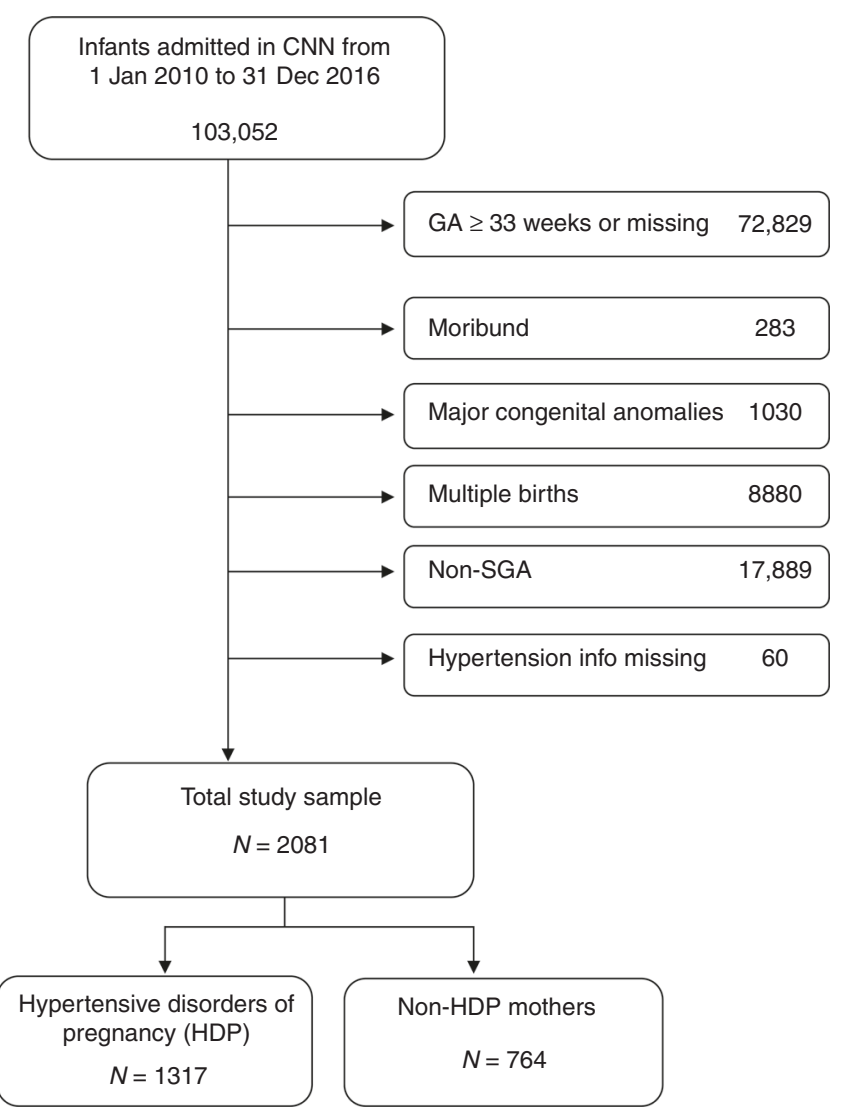

Fig. 1 Flow diagram of the study cohort

\section{DISCUSSION}

In this large national cohort of SGA infants $<33$ weeks gestation, we demonstrate that SGA infants of mothers with HDP had lower odds of mortality and BPD than SGA infants of non-HDP mothers. However, the lower odds of these outcomes were not consistent across gestational age groups. Mortality in SGA infants of HDP mothers was significantly lower in the 26-28 and 29-32 weeks gestation groups, but not in the $<26$ weeks gestation group. The odds of BPD was significantly lower in the 29-32 weeks gestation group with non-significant interaction between HDP and the gestational age groups. The odds of PDA was significantly lower in the $<26$ weeks gestation group. There was no difference in the

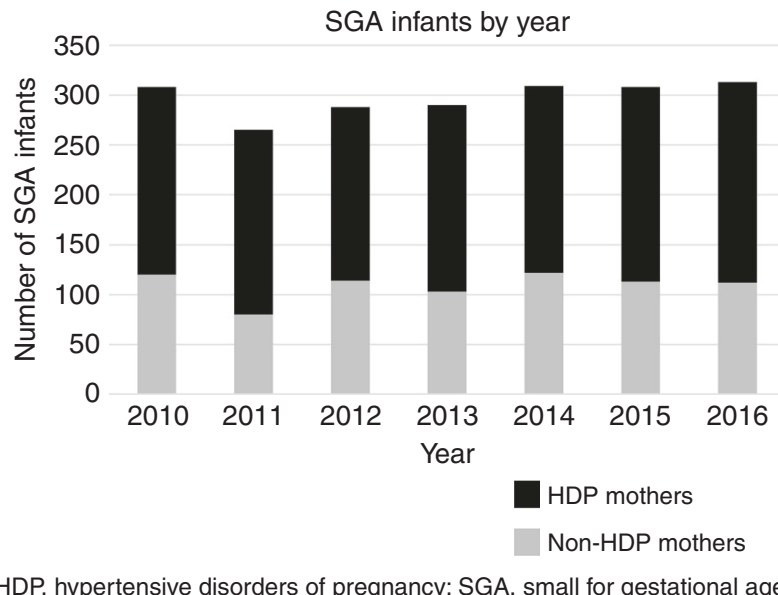

Fig. 2 Number of small for gestational age infants born during the study period

\begin{tabular}{|c|c|c|c|c|}
\hline Maternal age (years), mean (SD) & $31.2(5.8)$ & $30.6(5.9)$ & $31.6(5.8)$ & $<0.01$ \\
\hline GA (weeks), mean (SD) & $29.2(2.4)$ & $29.3(2.5)$ & $29.1(2.3)$ & 0.12 \\
\hline \multicolumn{5}{|l|}{ GA group } \\
\hline$<26$ weeks & $186(9 \%)$ & $79(10 \%)$ & $107(8 \%)$ & 0.03 \\
\hline Birth weight (gm), mean (SD) & $873(267)$ & $865(274)$ & $878(262)$ & 0.29 \\
\hline Birth head circumference $(\mathrm{cm})$, mean $(\mathrm{SD})$ & $25(2.7$ & $24.8(2.8)$ & $25.1(2.6)$ & 0.10 \\
\hline Gravida (>1) & $1240(60 \%)$ & $478(63 \%)$ & $762(58 \%)$ & 0.04 \\
\hline Substance use & $73(4 \%)$ & $45(6 \%)$ & $28(2 \%)$ & $<0.01$ \\
\hline Smoking & $256(12 \%)$ & $134(18 \%)$ & $122(9 \%)$ & $<0.01$ \\
\hline Chorioamnionitis & $84(5 \%)$ & $65(11 \%)$ & $19(2 \%)$ & $<0.01$ \\
\hline Cesarean birth & 1891 (91\%) & 647 (85\%) & 1244 (95\%) & $<0.01$ \\
\hline Male & $1123(54 \%)$ & $435(57 \%)$ & $688(52 \%)$ & 0.03 \\
\hline Out born & 211 (10\%) & $102(13 \%)$ & $109(8 \%)$ & $<0.01$ \\
\hline Apgar at $5 \mathrm{~min}<7$ & $539(26 \%)$ & $215(28 \%)$ & $324(25 \%)$ & 0.07 \\
\hline SNAP II score $>20$ & $320(16 \%)$ & $139(19 \%)$ & $181(14 \%)$ & $<0.01$ \\
\hline Surfactant use & 901 (43\%) & $305(40 \%)$ & 596 (45\%) & 0.01 \\
\hline
\end{tabular}


odds of IVH, severe ROP, NEC, and LOS between HDP and nonHDP groups across all gestational groups.

Regev et al. ${ }^{20}$ have reported on the outcomes of preterm SGA infants born to mothers with $\mathrm{PIH}$. Using the Israel Neonatal Network Database, they investigated the outcomes of SGA infants of 24-32 weeks gestation in the presence or absence of pregnancy complications such as prolonged rupture of membranes, chorioamnionitis, and antepartum hemorrhage. In the absence of pregnancy complications, infants of mothers with $\mathrm{PIH}$ had lower odds of NEC and higher odds of RDS. In the presence of pregnancy complications, infants of mothers with $\mathrm{PIH}$ had lower odds of severe IVH. There was no difference in mortality or BPD rates between infants of mothers with and without $\mathrm{PIH}$ irrespective of the presence of pregnancy complications. There are, however, significant differences between this study and ours, making comparison difficult. Their cohort included women with $\mathrm{PIH}$ only, defined as systolic blood pressure $>145 \mathrm{mmHg}$ and diastolic blood pressure $>95 \mathrm{mmHg}$ occurring after 20 weeks' gestation. It is unclear whether women with preeclampsia or chronic hypertension were included in the cohort. The numbers in the group with pregnancy complications was small, with 156 infants of PIH mothers and 512 without PIH. The outcomes are reported for the whole cohort and no gestational age-based subgroup analysis was described.

Our demonstration of lower odds of mortality corroborates two other studies that show reduced mortality in SGA infants of mothers with HDP. ${ }^{21,22}$ Using a large US-based database, Chen et al. ${ }^{21}$ reported lower odds of mortality in singleton SGA infants born to mothers with $\mathrm{PIH}$, which included gestational hypertension, preeclampsia, and eclampsia. The odds of mortality were lower for late preterm infants compared to early preterm infants,

Table 2. Comparison of neonatal outcomes for infants born at $<33$ weeks gestation to HDP and non-HDP mothers

\begin{tabular}{lccl}
\hline Characteristics & $\begin{array}{l}\text { HDP } \\
(N=1317)\end{array}$ & $\begin{array}{l}\text { Non-HDP } \\
(N=764)\end{array}$ & $\begin{array}{l}\text { Adjusted OR } \\
(95 \% \mathrm{Cl})\end{array}$ \\
\hline Mortality & $106(8 \%)$ & $99(13 \%)$ & $0.57(0.39,0.83)$ \\
BPD & $393(33 \%)$ & $247(37 \%)$ & $0.69(0.53,0.90)$ \\
Severe IVH & $58(5 \%)$ & $54(8 \%)$ & $0.78(0.50,1.21)$ \\
Severe ROP & $73(10 \%)$ & $41(10 \%)$ & $1.18(0.73,1.91)$ \\
NEC & $60(5 \%)$ & $44(6 \%)$ & $0.83(0.54,1.27)$ \\
LOS & $206(16 \%)$ & $127(17 \%)$ & $0.91(0.69,1.19)$ \\
PDA & $383(29 \%)$ & $216(29 \%)$ & $0.95(0.74,1.22)$ \\
\hline
\end{tabular}

${ }^{a}$ Adjusted for maternal age, any antenatal steroid use, maternal smoking gestational age, gender, cesarean section, out born status, and SNAP II scores $>20$

$H D P$ hypertensive disorders of pregnancy, $\mathrm{Cl}$ confidence intervals, $B P D$ bronchopulmonary dysplasia, $I V H$ intraventricular hemorrhage, $R O P$ retinopathy of prematurity, NEC necrotizing enterocolitis, LOS late-onset sepsis PDA patent ductus arteriosus, $O R$ odds ratio results similar to ours where the odds of mortality were lower in the higher gestational age groups. ${ }^{22}$ McBride et al. ${ }^{22}$ reported lower mortality in singleton preterm infants between 22 and 29 weeks gestation of mothers with HDP in the Vermont-Oxford Neonatal Network. Although investigators cite several reasons, the biological basis of the reduced mortality in SGA infants of mothers with HDP is unclear. Preterm SGA infants of mothers with HDP have higher levels of corticotrophin-releasing hormone, adrenocorticotrophin, and cortisol compared to infants of non-HDP mothers. $^{23}$ In addition, HDP mothers may be monitored more closely and several studies, including ours, have reported higher rates of antenatal corticosteroid use compared to non-HDP mothers who present acutely with pregnancy complications that lead to preterm delivery. ${ }^{20,22,24}$ Corticosteroids are known to decrease mortality in preterm infants. Some mothers with HDP may have increased utero-placental blood flow, as suggested by increased clearance of dehydroisoandrosterone. This increased flow may result in improved transfer of nutrients and oxygen to the fetus, which may result in improved survival. ${ }^{25}$ Lastly, the lower odds of mortality in preterm SGA infants of HDP mothers may not be related to HDP, but rather to increased mortality due to other causes of SGA or a higher rate of intrauterine death of SGA fetuses of HDP mothers. ${ }^{22}$

Although the odds of BPD were lower in the HDP group as a whole, on sub-group analysis there was lower odds only for the 29-32 weeks gestation group with insignificant interaction between HDP and the gestational age sub-groups. The association of BPD with HDP and SGA is, however, controversial, with studies reporting higher or lower risk and no difference. ${ }^{2,4,20,24,26}$ A large population-based study of very low birthweight infants demonstrated a lower incidence of BPD in mothers with preeclampsia, which was significant in the 31-34 weeks gestation group but not in earlier gestations, ${ }^{26}$ a result similar to ours. The investigators ascribed the reduced risk of BPD to lower levels of placental growth factor (PIGF) in preeclampsia, as higher levels of umbilical cord PIGF are reported in infants who develop BPD. ${ }^{27}$ PIGF knockout mice are resistant to elastase-induced emphysema, a condition that has similarities to BPD. ${ }^{28}$

Our results show no difference in odds of severe IVH in infants of HDP mothers in all the gestational age groups. Reports of severe IVH with HDP and SGA are also conflicting with lower and higher rates and no difference between infants of non-HDP vs. HDP mothers and between SGA and AGA infants. ${ }^{1,5,20,29}$ The reduced incidence of severe IVH in HDP and SGA is suspected to be due to higher antenatal steroid use. ${ }^{20}$

Rates of PDA were lower in the HDP group, but reached statistical significance only in infants $<26$ weeks gestation with significant interaction between HDP and gestational age subgroups. Boghossian et al. ${ }^{1}$ in a report on outcomes of SGA infants between 22 and 29 weeks gestation described lower rates of PDA in the lower gestational age groups. Lower rates of PDA are also reported in preeclampsia. ${ }^{30}$ The mechanism by which HDP and SGA reduce the incidence of PDA is unknown. The higher levels of corticosteroids in infants of mothers with HDP and the higher

Table 3. NICU resource use for SGA infants $<33$ weeks gestation born to HDP and non-HDP

\begin{tabular}{lccccc}
\hline Characteristics & Whole cohort $(N=2081)$ & HDP $(N=1317)$ & Non-HDP $(N=764)$ & $p$ Value & Adjusted mean difference $^{\text {a }}$ \\
\hline Duration of hospital stay, median (IQR) & $41(19,76)$ & $43(20,74)$ & $39(18,81)$ & 0.60 & $-4.6 \%(-12.9 \%, 4.5 \%)$ \\
Duration of oxygen use (IQR) & $3(0,32)$ & $3(0,31)$ & $3(0,34)$ & 0.89 & $-6.1 \%(-28.3 \%,-23 \%)$ \\
Duration of CPAP (IQR) & $4(0,18)$ & $5(1,20)$ & $3(0,15)$ & 0.003 & $20.5 \%(9.4 \%, 60.3 \%)$ \\
Duration of mechanical ventilation (IQR) & $1(0,8)$ & $1(0,8)$ & $1(0,9)$ & 0.50 & $-7.4 \%(-28.1,19.3 \%)$ \\
\hline
\end{tabular}

${ }^{a}$ Adjusted for maternal age, any antenatal steroid use, maternal smoking gestational age, gender, cesarean section, out born status, and SNAP II scores $>20$ IQR interquartile range, CPAP continuous positive airway pressure, HDP hypertensive disorders of pregnancy, NICU neonatal intensive care unit, SGA small for gestational age 


\begin{tabular}{|c|c|c|c|c|c|}
\hline Mortality & $<26$ & $50(47 \%)$ & 39 (49\%) & $1.09(0.57,2.08)$ & 0.009 \\
\hline \multirow[t]{3}{*}{ BPD } & $<26$ & 46 (84\%) & $36(86 \%)$ & $0.70(0.17,2.84)$ & 0.26 \\
\hline & $26-28$ & $214(63 \%)$ & $108(71 \%)$ & $0.74(0.48,1.15)$ & \\
\hline & $29-32$ & $133(16 \%)$ & 103 (22\%) & $0.57(0.42,0.79)$ & \\
\hline \multirow[t]{2}{*}{ Severe IVH } & $<26$ & 15 (17\%) & $19(26 \%)$ & $0.69(0.30,1.59)$ & 0.60 \\
\hline & $26-28$ & $25(7 \%)$ & $16(9 \%)$ & $0.71(0.36,1.38)$ & \\
\hline \multirow[t]{3}{*}{ NEC } & $<26$ & $9(9 \%)$ & $9(12 \%)$ & $0.55(0.19,1.57)$ & 0.35 \\
\hline & $26-28$ & $29(8 \%)$ & $16(9 \%)$ & $0.89(0.47,1.71)$ & \\
\hline & $29-32$ & $22(3 \%)$ & $19(4 \%)$ & $0.72(0.38,1.36)$ & \\
\hline \multirow[t]{3}{*}{ LOS } & $<26$ & $40(37 \%)$ & 32 (41\%) & $0.73(0.37,1.43)$ & 0.08 \\
\hline & $26-28$ & $102(27 \%)$ & $41(22 \%)$ & $1.23(0.80,1.89)$ & \\
\hline & $29-32$ & $64(8 \%)$ & $54(11 \%)$ & $0.69(0.47,1.04)$ & \\
\hline \multirow[t]{3}{*}{ PDA } & $<26$ & $69(68 \%)$ & $56(78 \%)$ & $0.42(0.19,0.95)$ & $<0.01$ \\
\hline & $26-28$ & 215 (56\%) & $91(50 \%)$ & $1.22(0.84,1.77)$ & \\
\hline & $29-32$ & 99 (12\%) & 69 (14\%) & $0.74(0.52,1.04)$ & \\
\hline
\end{tabular}

antenatal corticosteroid use may play a part, as corticosteroids are associated with a lower incidence of PDA. ${ }^{1}$ Moreover, both nitric oxide and carbon monoxide play a significant role in maintaining the patency of the ductus arteriosus. ${ }^{31}$ Lower levels of umbilical cord blood methemoglobin and carboxyhemoglobin, markers of nitric oxide and carbon monoxide levels, respectively, are described in preterm SGA infants and infants of preeclamptic mothers. ${ }^{32,33}$ However, more mechanistic and clinical studies are needed to fully explain this association in SGA infants of HDP mothers.

In our cohort, mortality and morbidity in the HDP group compared to the non-HDP group varied with gestational age, which has been previously reported. ${ }^{1,21,22}$ It is suggested that at earlier gestational ages, prematurity rather than SGA is the main factor that determines outcome. The higher mortality rate in infants of HDP mothers among earlier gestations may compete with the development of these morbidities. At later gestational ages, the presence of HDP, the cause of fetal growth restriction, may be responsible for the variation in mortality and morbidities. ${ }^{1}$ We attempted to evaluate these differential association by dividing our cohort in different gestational age stratums; however, despite having a large 7-year worth of national dataset, we need further studies to evaluate these associations by gestational ages.

Our results corroborate previously described associations of HDP and SGA with outcomes of preterm infants. The proportion of SGA infants in the HDP group in our cohort was $63 \%$, similar to other studies. ${ }^{1,4,22}$ Antenatal steroid use and C-section rates were higher and chorioamnionitis lower in the HDP group. ${ }^{20,22,24}$ Surfactant use was higher in the HDP group, suggesting more severe RDS. ${ }^{1,20}$ The antiangiogenic state in HDP contributes to surfactant dysfunction. ${ }^{34}$ The number of males was lower in the HDP group as reported in other studies. ${ }^{20,21}$ Although there is preponderance of males in preeclampsia, the ratio reverses the more preterm the delivery, with a preponderance of female fetuses. This has been attributed to male embryos being more susceptible to implantation disorder with some of those destined to develop preeclampsia resulting in spontaneous abortion. ${ }^{35}$

There are several strengths to our study. The CNN database is rigorously maintained with standardized data collection, which has been validated with a high degree of precision. The large population-based sample allowed us to adjust for several potential confounders, thereby improving confidence in results. As the cohort was population based, results can be interpreted at a population level, providing future data on associations that can be used to further develop a research agenda to explain the mechanistic pathways of these associations. Additionally, healthcare in Canada is universal, negating the effect of socioeconomic status and differing antenatal and postnatal care on the outcomes of preterm infants and resulting in a more diverse study population.

Nevertheless, the current study has limitations. We used a population-based birthweight reference to define SGA. Although controversial, some investigators have recommended the use of fetal growth standards or customized growth charts to define SGA. The use of fetal growth standards leads to a large number of infants being labeled as growth restricted without improving predictive models of outcome. Garite et al. ${ }^{5}$ demonstrated that growth restriction defined by fetal standards was associated with adverse outcome only when accompanied by neonatal birthweight less than the 10th percentile. Although customized growth 
charts consider maternal conditions that may affect fetal growth, they have not demonstrated improved identification of at risk newborns. ${ }^{36,37}$ Ehrenkranz ${ }^{38}$ has recommended that birthweightbased curves, rather than fetal weight-based standards, be used in clinical practice. Our data also does not differentiate between different forms of HDP. However, the majority of our patients were likely women with preeclampsia and as chronic hypertension is associated with increased risk of preeclampsia and SGA, the differentiation of HDP into its sub-types is not crucial. ${ }^{21,39}$ ShapiroMendoza et al. ${ }^{40}$ have recommended that in large multi-center databases, these conditions should not be separated because of inconsistencies in reporting across centers. In addition, the risk of selection intervention bias cannot be ruled out as HDP mothers and their infants may have been managed more intensely. We also did not have information on the use of magnesium sulfate and the severity of HDP.

In summary, the current study demonstrates that the lower odds of adverse outcomes in SGA infants of HDP mothers depend on gestational age. The entire cohort showed lower rates of mortality and BPD. On sub-group analysis based on gestational age, there was non-significant interaction between HDP and BPD. Reduced mortality continued to have significant interaction between HDP and the gestational age sub-groups. PDA had a lower incidence in the $<26$ weeks gestation HDP group with significant interaction between HDP and gestational age subgroups. In this era of precision medicine, our data can be used to identify preterm SGA infants at higher risk of adverse outcome and target them for specific interventions and therapies.

\section{ACKNOWLEDGEMENTS}

We gratefully acknowledge all site investigators and abstractors of the Canadian Neonatal Network (CNN) and Canadian Neonatal Follow-up Network (CNFUN). We also thank the staff at the Maternal-Infant Care (MiCare) Research Center at Mount Sinai Hospital, Toronto, ON, Canada for organizational support of the CNN, CNFUN, and this project. In addition, we thank Eugene W. Yoon, MSc, for statistical analysis assistance, as well as Sarah Hutchinson, PhD, and Iris Kulbatski, PhD, from MiCare for editorial assistance, in the preparation of this manuscript. MiCare is supported by a team grant from the Canadian Institutes of Health Research (CTP 87518), the Ontario Ministry of Health, and support from participating hospitals. P.S.S. holds an Applied Research Chair in Reproductive and Child Health Services and Policy Research awarded by the CIHR (APR-126340). The funding agencies had no role in the design and conduct of the study; collection, management, analysis, and interpretation of the data; preparation, review, or approval of the manuscript; and decision to submit the manuscript for publication.

\section{AUTHOR CONTRIBUTIONS}

K.Y.: Conception, design, interpretation, revised the manuscript, and finalized the manuscript. E.E.: Design, interpretation of data, and revised the manuscript. S.D.: Acquisition of data and revised the manuscript. W.Y.: Acquisition of data and revised the manuscript. E.P.: Acquisition of data and revised manuscript. R.C.: Acquisition of data and revised the manuscript. P.S.S.: Analysis of data, interpretation of data, revised manuscript, and final approval of the manuscript.

\section{ADDITIONAL INFORMATION}

The online version of this article (https://doi.org/10.1038/s41390-019-0416-6) contains supplementary material, which is available to authorized users.

Competing interests: The authors declare no competing interests.

Publisher's note: Springer Nature remains neutral with regard to jurisdictional claims in published maps and institutional affiliations.

\section{REFERENCES}

1. Boghossian, N. S., Geraci M., Edwards E. M. \& Horbar J. D. Morbidity and mortality in small for gestational age infants at 22 to 29 weeks' gestation. Pediatrics 141, e20172533 (2018).
2. De Jesus, L. C. et al. Outcomes of small for gestational age infants born at $<27$ weeks' gestation. J. Pediatr. 163, 55-60 (2013)

3. Malhotra, A. et al. Neonatal morbidities of fetal growth restriction: pathophysiology and impact. Front. Endocrinol. (Lausanne) 10, 55 (2019).

4. Zeitlin, J. et al. Impact of fetal growth restriction on mortality and morbidity in a very preterm birth cohort. J. Pediatr. 157, 733-739 (2010).

5. Garite, T. J., Clark, R. \& Thorp, J. A. Intrauterine growth restriction increases morbidity and mortality among premature neonates. Am. J. Obstet. Gynecol. 191, 481-487 (2004).

6. Tsai, L. Y., Chen, Y. L., Tsou, K. I. \& Mu, S. C. Taiwan Premature Infant Developmental Collaborative Study Group. The impact of small-for-gestational-age on neonatal outcome among very-low-birth-weight infants. Pediatr. Neonatol. 56, 101-107 (2015).

7. Kiechl-Kohlendorfer, U., Ralser, E., Pupp Peglow, U., Reiter, G. \& Trawoger, R. Adverse neurodevelopmental outcome in preterm infants: risk factor profiles for different gestational ages. Acta Paediatr. 98, 792-796 (2009).

8. Nardozza, L. M. et al. Fetal growth restriction: current knowledge. Arch. Gynecol. Obstet. 295, 1061-1077 (2017).

9. Hutcheon, J. A., Lisonkova, S. \& Joseph, K. S. Epidemiology of pre-eclampsia and the other hypertensive disorders of pregnancy. Best Pract. Res. Clin. Obstet. Gynaecol. 25, 391-403 (2011).

10. Gemmell, L. et al. Hypertensive disorders of pregnancy and outcomes of preterm infants of 24 to 28 weeks' gestation. J. Perinatol. 36, 1067-1072 (2016).

11. LMSS, Rugolo \& Bentlin, M. R. CEP T preeclampsia: early and late neonatal outcomes. NeoReviews 13, e532-e541 (2012).

12. Lee, S. K. et al. Variations in practice and outcomes in the Canadian NICU network: 1996-1997. Pediatrics 106, 1070-1079 (2000).

13. Shah, P. S. et al. Internal udit of the Canadian Neonatal Network Data Collection System. Am. J. Perinatol. 34, 1241-1249 (2017).

14. The Canadian Neonatal Network. Abstractor" Manual v.3.3.0 (The Canadian Neonatal Network, Toronto, Canada, 2018).

15. Kramer, M. S. et al. A new and improved population-based Canadian reference for birth weight for gestational age. Pediatrics 108, E35 (2001).

16. Shennan, A. T., Dunn, M. S., Ohlsson, A., Lennox, K. \& Hoskins, E. M. Abnormal pulmonary outcomes in premature infants: prediction from oxygen requirement in the neonatal period. Pediatrics 82, 527-532 (1988).

17. Papile, L. A., Munsick-Bruno, G. \& Schaefer, A. Relationship of cerebral intraventricular hemorrhage and early childhood neurologic handicaps. J. Pediatr. 103, 273-277 (1983).

18. Bell, M. J. et al. Neonatal necrotizing enterocolitis-therapeutic decisions based upon clinical staging. Ann. Surg. 187, 1-7 (1978).

19. Gole, G. et al. The International Classification of Retinopathy of Prematurity Revisited. Arch. Ophathlmol. 123, 991-999 (2005).

20. Regev R. H. et al. Outcome of singleton preterm small for gestational age infants born to mothers with pregnancy-induced hypertension. A population-based study. J. Matern. Fetal Neonatal Med. 28, 666-73 (2015).

21. Chen, X. K., Wen, S. W., Smith, G., Yang, Q. \& Walker, M. Pregnancy-induced hypertension and infant mortality: Roles of birthweight centiles and gestational age. BJOG 114, 24-31 (2007).

22. McBride, C. A., Bernstein, I. M., Badger, G. J. \& Soll, R. F. Maternal hypertension and mortality in small for gestational age 22- to 29-week infants. Reprod. Sci. 25, 276-280 (2018).

23. Goland, R. S. et al. Elevated levels of umbilical cord plasma corticotropin-releasing hormone in growth-retarded fetuses. J. Clin. Endocrinol. Metab. 77, 1174-1179 (1993).

24. Soliman, N., Chaput, K., Alshaikh, B. \& Yusuf, K. Preeclampsia and the risk of bronchopulmonary dysplasia in preterm infants less than 32 weeks' gestation. Am. J. Perinatol. 34, 585-592 (2017).

25. Gant, N. F., Hutchinson, H. T., Siiteri, P. K. \& MacDonald, P. C. Study of the metabolic clearance rate of dehydroisoandrosterone sulfate in pregnancy. Am. J. Obstet. Gynecol. 111, 555-563 (1971).

26. Yen, T. A. et al. Preeclampsia and the risk of bronchopulmonary dysplasia in VLBW infants: a population based study. PLOS ONE 8, e75168 (2013).

27. Tsao, P. N. et al. Placenta growth factor elevation in the cord blood of premature neonates predicts poor pulmonary outcome. Pediatrics 113, 1348-1351 (2004).

28. Cheng, S. L. et al. Prevention of elastase-induced emphysema in placenta growth factor knock-out mice. Respir. Res. 10, 115 (2009).

29. Chevallier, M. et al. Leading causes of preterm delivery as risk factors for intraventricular hemorrhage in very preterm infants: results of the EPIPAGE 2 cohort study. Am. J. Obstet. Gynecol. 216, 518 e511-518 e512 (2017).

30. Huang, H. C. et al. Preeclampsia and retinopathy of prematurity in very-low-birthweight infants: a population-based study. PLOS ONE 10, e0143248 (2015).

31. Hung, Y. C., Yeh, J. L. \& Hsu, J. H. Molecular mechanisms for regulating postnatal ductus arteriosus closure. Int. J. Mol. Sci. 19, E1861 (2018). 
32. Yusuf, K., Kamaluddeen, M., Wilson, R. D. \& Akierman, A. Carboxyhemoglobin levels in umbilical cord blood of women with pre-eclampsia and intrauterine growth restriction. J. Perinat. Med. 40, 619-624 (2012).

33. Yusuf, K. et al. Methemoglobin levels in umbilical cord blood of women with intrauterine growth restriction and preeclampsia. J. Matern. Fetal Neonatal Med. 27, 789-794 (2014).

34. Wang, A. et al. Circulating anti-angiogenic factors during hypertensive pregnancy and increased risk of respiratory distress syndrome in preterm neonates. J. Matern. Fetal Neonatal Med. 25, 1447-1452 (2012).

35. Jaskolka, D., Retnakaran, R., Zinman, B. \& Kramer, C. K. Fetal sex and maternal risk of pre-eclampsia/eclampsia: a systematic review and meta-analysis. BJOG 124, 553-560 (2017)
36. Larkin, J. C., Hill, L. M., Speer, P. D. \& Simhan, H. N. Risk of morbid perinatal outcomes in small-for-gestational-age pregnancies: customized compared with conventional standards of fetal growth. Obstet. Gynecol. 119, 21-27 (2012).

37. Mayer, C. \& Joseph, K. S. Fetal growth: a review of terms, concepts and issues relevant to obstetrics. Ultrasound Obstet. Gynecol. 41, 136-145 (2013).

38. Ehrenkranz, R. A. Estimated fetal weights versus birth weights: should the reference intrauterine growth curves based on birth weights be retired? Arch. Dis. Child Fetal Neonatal Ed. 92, F161-F162 (2007).

39. von Dadelszen, P. \& Magee, L. A. Maternal hypertension and neonatal outcome among small for gestational age infants. Obstet. Gynecol. 106, 1416-1417 (2005).

40. Shapiro-Mendoza, C. K. et al. Effect of late-preterm birth and maternal medical conditions on newborn morbidity risk. Pediatrics 121, e223-e232 (2008). 